\title{
The effects of gelled konjac glucomannan fibre on appetite and energy intake in healthy individuals: a randomised cross-over trial
}

\author{
Fei Au-Yeung ${ }^{1,2}$, Elena Jovanovski ${ }^{1,2}$, Alexandra L. Jenkins ${ }^{1}$, Andreea Zurbau ${ }^{1,2}$, Hoang V. T. Ho ${ }^{1}$ and \\ Vladimir Vuksan ${ }^{1,2,3,4,5 *}$ \\ ${ }^{1}$ Clinical Nutrition and Risk Factor Modification Centre, St. Michael's Hospital, Toronto, ON M5B 1W8, Canada \\ ${ }^{2}$ Department of Nutritional Sciences, Faculty of Medicine, University of Toronto, Toronto, ON M5S 3E2, Canada \\ ${ }^{3}$ Department of Medicine, Faculty of Medicine, University of Toronto, Toronto, ON M5S 1A8, Canada \\ ${ }^{4}$ Keenan Research Centre of the Li Ka Shing Knowledge Institute, St. Michael's Hospital, Toronto, ON M5B 1T8, Canada \\ ${ }^{5}$ Division of Endocrinology \& Metabolism, St. Michael's Hospital, Toronto, ON M5B 1W8, Canada \\ (Submitted 13 April 2017 - Final revision received 23 October 2017 - Accepted 24 October 2017-First published online 5 December 2017)
}

\section{Abstract}

Konjac glucomannan (KGM) is a viscous dietary fibre that can form a solid, low-energy gel when hydrated and is commonly consumed in a noodle form (KGM-gel). Increased meal viscosity from gel-forming fibres have been associated with prolonged satiety, but no studies to date have evaluated this effect with KGM-gel. Thus, our objective was to evaluate subsequent food intake and satiety of KGM-gel noodles when replacing a high-carbohydrate preload, in a dose-response manner. Utilising a randomised, controlled, cross-over design, sixteen healthy individuals (twelve females/four males; age: $26 \cdot 0$ (sD 11.8) years; BMI: $23 \cdot 1$ (sD 3.2) kg/m²) received 325 ml volume-matched preloads of three KGM-gel noodle substitution levels: (i) all pasta with no KGM-gel (1849 kJ (442 kcal), control), half pasta and half KGM-gel (1084 kJ (259 kcal), 50-KGM) or no pasta and all KGM-gel (322 kJ (77 kcal), 100-KGM). Satiety was assessed over 90 min followed by an ad libitum dessert. Compared with control, cumulative energy intake was $47 \%(-1761 \mathrm{~kJ}(-421 \mathrm{kcal}))$ and $23 \%(-841 \mathrm{~kJ}(-201 \mathrm{kcal}))$ lower for $100-\mathrm{KGM}$ and 50-KGM, respectively (both $P<0 \cdot 001$ ), but no differences in subsequent energy intake was observed. Ratings of hunger were $31 \%$ higher $(P=0.03)$ for 100 -KGM when compared with control, and were $19 \%$ lower $(P=0.04)$ for fullness and $28 \%$ higher $(P=0.04)$ for prospective consumption when comparing 100-KGM to 50-KGM. Palatability was similar across all treatments. Replacement of a high-carbohydrate preload with low-energy KGM-gel noodles did not promote additional food intake despite large differences in energy. The energy deficit incurred from partial KGM-gel substitution may have relevance in weight loss regimens, and should be further evaluated beyond the healthy population.

Key words: Randomised controlled trials: Dietary fibres: Konjac glucomannan: Appetite: Energy intakes

Sustained overeating and physical inactivity often leads to increased body weight. Despite numerous advances in dietary and lifestyle approaches, body weight regulation remains challenging. One major obstacle when adhering to an energyrestricted diet is to endure hunger, a predictor of success for long term weight loss and maintenance ${ }^{(1,2)}$.

Several promising approaches for satiety regulation have emerged, including the consumption of low energy density (ED) foods, increased intake of dietary fibre, and by manipulation of the food matrix, such as the physical form or viscosity of the meal ${ }^{(3-5)}$. Although several foods have been identified to possess some of these characteristics, few alternatives are available when replacing high ED foods in the diet without any major dietary modifications. An emerging novel food that appears to embody all three satiety approaches are low-energy foods made from a dietary fibre known as konjac glucomannan (KGM).

KGM is a highly viscous dietary fibre derived from the perennial plant Amorphophallus konjac and has a long history of use as a traditional food in Eastern $\mathrm{Asia}^{(6)}$. At low concentrations, KGM is capable of forming a strong gel (KGM-gel) that is approximately $97 \%$ water when in the presence of a coagulant, representing one of the lowest ED (approximately $0.25 \mathrm{~kJ} / \mathrm{g}$ $(0.06 \mathrm{kcal} / \mathrm{g})$ foods available ${ }^{(7)}$. KGM-gel has a neutral taste and can be formed into various food shapes such as long, noodle-like strands popularly known as 'shirataki noodles'. This can provide a potential method to reduce energy intake with minimal effort when replacing similar shaped foods that are high in ED without changing the volume or sensory characteristics of the meal. In addition, KGM is resistant to typical gastric digestion like most

Abbreviations: CS, composite score; ED, energy density; KGM, Konjac glucomannan; SQ, satiety quotient; VAS, visual analogue scales.

* Corresponding author: V. Vuksan, fax +416864 5538, email v.vuksan@utoronto.ca 
fibres due to the $\beta$-1,4-linkages within the fibre chain that cannot be hydrolysed by salivary or pancreatic amylase. Another feature of KGM-gels is its high firmness, which has been estimated to be between $0.8-1.6 \mathrm{~N}$, which exceeds the strength typically exerted by the stomach during digestion ${ }^{(8,9)}$. This additional property results in KGM-gels being retained longer in the stomach, and may be a mechanism that independently promotes satiety responses to reduce energy intake.

Despite these favourable characteristics and increasing public interest for low-energy foods that are satiety-enhancing, no study to date has investigated the role of KGM-gel foods and its effect on energy intake and satiety in a controlled clinical setting. It is also unclear whether the energy deficit incurred from substituting high ED foods with KGM-gel foods will be compensated at a subsequent meal, abolishing its effectiveness as a potential weight management tool. Therefore, we sought to evaluate the effects of substituting KGM-gel shirataki noodles administrated at two levels in place of a high-carbohydrate preload of equal volume, but different energy contents, on subsequent food intake and subjective satiety in healthy individuals. We hypothesised that increasing KGM-gel noodles would lead to greater energy compensation in a dose-dependent manner at the subsequent meal due to the large differences in energy and macronutrient content between preloads.

\section{Methods}

\section{Participants}

Healthy participants were recruited into the study by advertisement flyers placed on the campus of the University of Toronto and in St. Michael's Hospital, Toronto, Canada. Individuals who responded to the advertisements were interviewed by telephone to ensure they met the initial criteria for inclusion into the study: age 18-65 years, BMI between 18 and $25 \mathrm{~kg} / \mathrm{m}^{2}$, no presence or known history of major diseases, not using prescription medication and/or natural health products during the study period, not pregnant or lactating, no known food allergies and weight stable for the past 2 months.

Potential participants meeting the initial criteria were invited to the clinic, where anthropometrics (height, weight, \% body fat) were taken, and several questionnaires were completed regarding dietary and lifestyle regimens. All participants gave informed written consent before participating and conducted according to the Declaration of Helsinki. The study was approved by the St. Michael's Hospital Research Ethics Board and was conducted at the Risk Factor Modification Centre, St. Michael's Hospital (Toronto, Canada). The trial protocol was registered with ClinicalTrials.gov, identifier NCT01875627.

\section{Design}

In a randomised, single-blinded, controlled, dose-response, cross-over design, participants were administered one of three intervention preloads in a randomised sequence determined by a random number table generated by a statistician. Investigators, study personnel and statisticians were blinded to intervention preloads. Participants visited the clinic between
08.00 and 10.00 hours after a $10-12 \mathrm{~h}$ overnight fast on three separate occasions separated by a washout period of at least $2 \mathrm{~d}$. Upon arrival at the clinic, anthropometric data were collected and participants were seated in a quiet, isolated area for the remainder of the visit. Participants were told to maintain their usual dietary and lifestyle regimens during the study period and this was assessed by a questionnaire. Baseline (0 min) satiety and symptoms were recorded and the preloads were served subsequently. Participants rated the palatability of the preload immediately after consumption and satiety and symptoms questionnaires were completed at 15, 30, 45, 60, 75 and $90 \mathrm{~min}$ after the first bite of the preload. Participants were given 15 min to consume the preload and water served. Participants were only allowed to consume the foods given to them at the clinic. Immediately after completing the satiety and symptoms questionnaires at $90 \mathrm{~min}$, participants were offered an ad libitum dessert. Dessert was served in $150 \mathrm{~g}$ portions and participants were told to consume as much of the dessert as they desired until they felt comfortably full. Preload and dessert meals were served with ad libitum water in portions of $240 \mathrm{ml}$ and the same amount of water was served with the respective meal during subsequent visits for each participant. All food and drinks served were weighed using a digital scale to the nearest $0 \cdot 1 \mathrm{~g}$ before and after serving to determine the intake of food.

\section{Study materials}

Macronutrient composition and characteristics of the preloads are described in Table 1. The intervention preloads were the following: (1) cooked pasta noodles with no KGM-gel noodles (control); (2) substitution of half cooked pasta noodles with KGM-gel noodles (50-KGM); (3) complete substitution of cooked pasta noodles with KGM-gel noodles (100-KGM). Preloads were matched for volume and appearance, as they were mixed with the same quantity of pasta sauce before serving. All preloads were cooked and prepared on the same day as the clinical visit by a trained research personnel independent of the study to maintain blinding. The ad libitum dessert consisted of bite-sized (approximately $2.5 \mathrm{~cm}$ ) pieces of hazelnut-flavoured wafer cookies (Quadratini Wafer Cookies; Loaker $\left.{ }^{\circ}\right)$ that contains $21.63 \mathrm{~kJ} / \mathrm{g}(5.17 \mathrm{kcal} / \mathrm{g})$ (48\% carbohydrate, $47 \%$ fat, $5 \%$ protein). All study materials were commercially available food products purchased from a local supermarket and were prepared in accordance with the instructions found on the manufacturer's label.

\section{Measurements}

Participants were asked to remove any heavy clothing before collecting anthropometrics. Body weight was collected using a digital scale (TANITA) to ensure no major changes in anthropometrics occurred between visits. If major changes occurred, the participant was excluded from the study. Height was measured using a wall mounted stadiometer.

Participants completed satiety questionnaires in the form of $100 \mathrm{~mm}$ visual analogue scales (VAS) assessing four subjective satiety measurements: desire to eat, hunger, fullness and prospective consumption. Each VAS were anchored on both sides with descriptors adapted from Blundell et $a l{ }^{(10)}$. The satiety 
Table 1. Composition and characteristics of the preloads

\begin{tabular}{|c|c|c|c|}
\hline & \multicolumn{3}{|c|}{ Preloads } \\
\hline & Control & 50-KGM & 100-KGM \\
\hline \multicolumn{4}{|l|}{ Ingredients } \\
\hline Cooked pasta noodles $(\mathrm{g})^{\star}$ & $220 \cdot 0$ & $110 \cdot 0$ & 0.0 \\
\hline Konjac-gel noodles $(\mathrm{g}) \dagger$ & 0.0 & 122.5 & $240 \cdot 0$ \\
\hline Pasta sauce $(\mathrm{g}) \ddagger$ & $80 \cdot 0$ & $80 \cdot 0$ & $80 \cdot 0$ \\
\hline \multicolumn{4}{|l|}{ Nutrients§ } \\
\hline Energy content (kJ) & $1847 \cdot 2$ & 1084.5 & 321.3 \\
\hline Energy content (kcal) & 441.5 & $259 \cdot 2$ & $76 \cdot 8$ \\
\hline Energy density $(\mathrm{kJ} / \mathrm{g})$ & $6 \cdot 2$ & 3.5 & 1.0 \\
\hline Available carbohydrates (g) & 80.5 & 43.5 & $6 \cdot 4$ \\
\hline Protein $(\mathrm{g})$ & 14.9 & 8.4 & 1.9 \\
\hline Fat $(\mathrm{g})$ & 6.9 & $6 \cdot 0$ & $5 \cdot 1$ \\
\hline Total dietary fibre $(\mathrm{g})$ & 6.5 & $7 \cdot 3$ & $8 \cdot 0$ \\
\hline Fibre from Konjac $(\mathrm{g})$ & 0.0 & 4.0 & 8.0 \\
\hline Weight (g) & $300 \cdot 0$ & 312.5 & $320 \cdot 0$ \\
\hline Volume (ml) & $325 \cdot 0$ & $325 \cdot 0$ & $325 \cdot 0$ \\
\hline
\end{tabular}

recorded before serving the preload were used as baseline ( $0 \mathrm{~min}$ ) measurements and satiety after the first bite of the preload were recorded every $15 \mathrm{~min}$ thereafter until $90 \mathrm{~min}$. Response of satiety to the preloads were presented as mean satiety and evaluated by calculating the total AUC after 90 min using the trapezoid method ${ }^{(11)}$.

To investigate the efficiency and capacity of the preload to influence satiety, the satiety quotient (SQ) was calculated for each satiety measurement, except for sensations of fullness, at each postprandial time measured following the equation adapted from Green et $a l .{ }^{(12)}: \mathrm{SQ}(\mathrm{mm} / \mathrm{kJ})=($ baseline satiety $(\mathrm{mm})$ - postprandial satiety $(\mathrm{mm})) /($ energy content of preload $(\mathrm{kJ}))$. Fullness used a reversed SQ where (postprandial satiety $(\mathrm{mm})$-baseline satiety $(\mathrm{mm})$ ) was used instead to facilitate the comparison between the other satiety measurements. A higher SQ for each satiety measurement would represent a greater satiety response and a lower SQ would represent a weaker satiety response because the SQ accounts for baseline satiety and the energy content of the preload.

Gastrointestinal symptoms of bloating, belching, diarrhoea, flatulence and nausea were reported by presence of absence of the symptom at each time interval satiety were measured. If present, severity was recorded using a $100 \mathrm{~mm}$ VAS. VAS were anchored with the descriptors 'low' and 'high' on the left and right ends of the VAS, respectively.

Preload palatability was measured using a $100 \mathrm{~mm}$ VAS immediately after consumption of the preload anchored with the phrases 'very unpalatable' to 'very palatable' on the left and right ends of the VAS, respectively.

\section{Statistical analysis}

The primary outcome measured was energy compensation defined as the energy intake $90 \mathrm{~min}$ after preload administration. Total food intake ( $\mathrm{g}$ and $\mathrm{kJ}$ ), satiety (baseline, $90 \mathrm{~min}$ AUC and SQ), palatability ratings and gastrointestinal symptoms were evaluated as secondary outcomes. A composite score (CS) for satiety adapted from a previous study ${ }^{(13)}$ was calculated to reflect the overall effect of the preload on satiety using each satiety measurement: CS $(\mathrm{mm})=($ hunger + desire to eat $+(100-$ fullness $)+$ prospective consumption $) / 4$.

Data are presented as means with their standard errors unless otherwise specified. All statistical procedures were performed using the Statistical Analysis System software package, University Edition (SAS Institute Inc.). Results were considered significant at $P<0.05$. Data normality was assessed visually and using the Shapiro-Wilk procedure. The effect of the interventions on satiety was assessed using a mixed model ANOVA (proc mixed; SAS) with preload, time, and time $\times$ preload interaction as fixed factors and participants as the repeated factor. Pairwise analyses were conducted to assess the differences between interventions and adjusted for multiple comparisons using the Tukey-Kramer method for multiple comparisons. Mean satiety, AUC for satiety, mean SQ, time to eat, palatability, subsequent energy intake and cumulative energy intake were assessed with a similar procedure without time and its interactive factor. For all satiety measures, baseline fasting $(0 \mathrm{~min})$ values were included as a covariate to adjust for the variability between intervention days and individuals. For symptoms, data were grouped by presence during the clinical visit and assessed using a $\chi^{2}$ test.

The power calculation was based on a $628 \mathrm{~kJ}$ ( $150 \mathrm{kcal})$ difference between groups in a two-tailed test with a significance level of 0.05 and a power of $80 \%$. Based on a previous study of fibres and food intake, sixteen individuals were to be recruited into the study ${ }^{(14)}$.

\section{Results}

\section{Participants}

In all, sixteen participants (twelve females: four males) with mean age of 26.0 (SD 11.8) years (range: 18-62 years) and BMI of $23 \cdot 1$ (SD $3 \cdot 2) \mathrm{kg} / \mathrm{m}^{2}$ were enrolled in the study. All participants completed the study and were compliant with the study protocols. No significant changes in body weight or 
body fat occurred over the duration of the study (data not shown). Participants did not report any significant changes to diet or physical activity levels during the study period.

\section{Energy intake}

Despite significant energy differences of the preloads, energy intakes during dessert 90 min later were similar between interventions (Fig. 1, effect of treatment: $P=0 \cdot 71)$. Subsequent energy intake was 1534.7 (SEM 142) kJ (366.8 (SEM 34) kcal) for control, $1631 \cdot 8$ (SEM 142) kJ (390.1 (SEM 34) kcal) for 50-KGM and $1652 \cdot 3$ (SEM 142) kJ (394.9 (SEM 34) kcal) for 100-KGM and subsequent food intake was 70.3 (SEM 7) $\mathrm{g}$ for control, 74.7 (SEM 7) $\mathrm{g}$ for 50-KGM and 75.7 (SEM 7) g for 100-KGM. No differences in subsequent energy intake (100-KGM $v$. control: $117 \cdot 9(150 \cdot 2) \mathrm{kJ}$ (28.2 (sEm 35.9) kcal), $P=0 \cdot 72 ; 50-K G M v$. control: $97 \cdot 9(150 \cdot 2) \mathrm{kJ}$ (23.4 (SEM 35.9) kcal), $P=0.79 ; 100-K G M \quad v$. 50-KGM: $20 \cdot 0$ $(150.2) \mathrm{kJ}$ (4.8 (SEM 35.9) kcal), $P=0.99)$ or food intake (100-KGM $v$. control: 5.4 (SEM 6.9) g, $P=0.72 ; 50-\mathrm{KGM} \quad v$. control: 4.5 (SEM 6.9) g, $P=0.79 ; 100-K G M \quad v$. 50-KGM: 0.9 (SEM 6.9) g, $P=0.99)$ at $90 \mathrm{~min}$ was observed. Cumulative energy intake across the two meals (preload and dessert) remained significantly different for 50-KGM (-841 (sem 151) kJ (-201 (sEM 36) kcal), $P<0 \cdot 01)$ and 100-KGM (-1761 (sem 151) kJ (-421 (sEM 36) kcal), $P<0.01)$ relative to control and when comparing $100-\mathrm{KGM}$ to 50-KGM (-946.3 (sem 150.2) kJ (-219.8 (sEm 35.9) kcal), $P<0 \cdot 01)$.

\section{Satiety}

Mean satiety outcomes are presented in Table 2 . A significant effect of time $(P<0.001)$ and preload $(P<0.001)$ was observed for all satiety measures, but no interaction effect was observed. Mean ratings for desire to eat were not significantly different across comparisons (100-KGM $v$. control: 8.5 (SEM 4.0) $\mathrm{mm}, P=0 \cdot 10$; 50-KGM $v$. control: 0.1 (SEM 4.0) mm, $P=0.99$; 100-KGM $v$. 50-KGM: 8.5 (SEM 3.9), $P=0.09$ ). Mean ratings for hunger was significantly higher when comparing $100-\mathrm{KGM}$ to control $(10 \cdot 0$ (SEM 3.9) $\mathrm{mm}, P=0.04$ ) and not significantly different for other comparisons (100-KGM to 50-KGM: $9 \cdot 0$ (SEM 3.7) mm, $P=0.05$; 50-KGM to control: $1.0(\operatorname{SEM} 3.7) \mathrm{mm}, P=0.96$ ). Mean ratings for

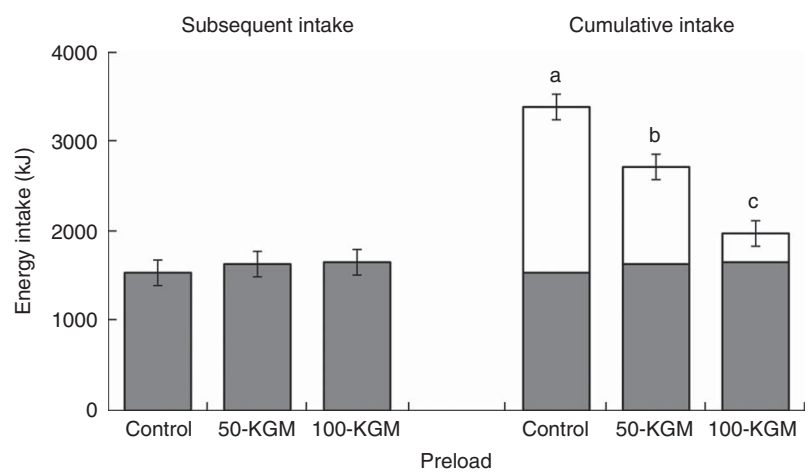

Fig. 1. Subsequent energy intake at $90 \mathrm{~min}$ after preload administration. Cumulative intake was defined as the total energy intake from the preload and the subsequent meal. Values are means with their standard errors represented by vertical bars. $\square$, Preload; $\square$, dessert; KGM, konjac glucomannan. ${ }^{\text {a,b,c }}$ Preloads with unlike letters were significantly different $(P<0.05$, Tukey adjusted). fullness were not significantly different across comparisons (100-KGM $v$. control: -8.7 (SEM 4.1) mm, $P=0 \cdot 10$; 50-KGM $v$. control: $1 \cdot 4(\mathrm{SEM} 4 \cdot 1) \mathrm{mm}, P=0 \cdot 94 ; 100-\mathrm{KGM} v$. 50-KGM: $-10 \cdot 2$ (SEM 4.2), $P=0 \cdot 06$ ). Mean ratings for prospective food consumption was significantly lower when comparing 50-KGM to 100-KGM $(-9.1$ (SEM 3.4) mm, $P=0.03$ ) and not for other comparisons (100-KGM $v$. control: 7.4 (SEM 3.4) mm, $P=0 \cdot 10$; 50-KGM $v$. control: 1.7 (SEM 3.5$) \mathrm{mm}, P=0.87$ ). Mean CS were not significantly different across comparisons (100-KGM $v$. control: 8.2 (SEM 3.7) mm, $P=0 \cdot 09$; 50-KGM $v$. control: $-0.4(\operatorname{sem} 3.7) \mathrm{mm}, P=0.99$; 100-KGM $v$. 50-KGM: $8 \cdot 6$ (SEM 3.6), $P=0 \cdot 06$ ).

When comparing the AUC for satiety measurements, no significant differences were observed for desire to eat (100-KGM v. control: $786.4(\mathrm{SEM} 374.9) \mathrm{mm} \times \mathrm{min}^{-1}, P=0 \cdot 11$; 50-KGM $v$. control: -9.9 (SEM 374.6$) \mathrm{mm} \times \mathrm{min}^{-1}, \quad P=0.99$; 100-KGM $v$. 50-KGM: 796.2 (SEM 371.0) $\mathrm{mm} \times \mathrm{min}^{-1}, P=0 \cdot 10$ ). For hunger, only 100-KGM compared with control increased hunger (100-KGM $v$. control: 945.6 (SEM 358.8) $\mathrm{mm} \times \mathrm{min}^{-1}, \quad P=0.03$; 50-KGM $v$. control: $100 \cdot 1$ (SEM 346.9) $\mathrm{mm} \times \mathrm{min}^{-1}, \quad P=0.96$; 100-KGM $v$. 50-KGM: 845.5 (SEM 344.4) $\mathrm{mm} \times \mathrm{min}^{-1}, P=0.05$ ). Fullness decreased only when comparing 100-KGM to 50-KGM (100-KGM $v$. 50-KGM: -1005.5 (SEM 394.7) $\mathrm{mm} \times \mathrm{min}^{-1}, P=0.04$; 100-KGM $v$. control: -833.8 (SEM 389.2) $\mathrm{mm} \times \min ^{-1}, P=0 \cdot 10$; 50-KGM $v$. control: 171.7 (SEM 384.0) $\mathrm{mm} \times \mathrm{min}^{-1}, P=0.90$ ) and prospective food consumption increased when comparing 100-KGM to 50-KGM (100-KGM v. 50-KGM: $862 \cdot 4$ (SEM 321.7 ) $\mathrm{mm} \times \mathrm{min}^{-1}, \quad P=0.03 ; 100-\mathrm{KGM} \quad v$. control: $679 \cdot 6$ (SEM $320 \cdot 3) \mathrm{mm} \times \mathrm{min}^{-1}, \quad P=0 \cdot 10$; 50-KGM $v$. control: $-182 \cdot 7$ (SEM 323.7$) \mathrm{mm} \times \mathrm{min}^{-1}, \quad P=0 \cdot 84$ ). No significant differences were detected for CS AUC (100-KGM $v$. control: $769 \cdot 6$ (SEM 348.3) $\mathrm{mm} \times \mathrm{min}^{-1}, \quad P=0.09 ; \quad 50-\mathrm{KGM} \quad v$. control: -49.9 (SEM 343.3) $\mathrm{mm} \times \mathrm{min}^{-1}, P=0.99 ; 100-\mathrm{KGM} \quad v$. 50-KGM: 819.5 (sEM 341.4) $\mathrm{mm} \times \mathrm{min}^{-1}, P=0.06$ ).

The satiating capacity of each preload was also assessed with the SQ, where changes in satiety ratings were expressed as a factor of energy intake in Table 2 . The mean SQ was significantly higher for 100-KGM for all satiety measurements when compared with both 50-KGM and control preloads $(P<0 \cdot 0001)$ and no differences were detected among other measurements between preloads. For desire to eat, the differences in SQ were 0.125 (SEM 0.0096$) \mathrm{mm} / \mathrm{kJ}(P<0 \cdot 01)$ for $100-\mathrm{KGM} v$. control, 0.0134 (sEM 0.0096$) \mathrm{mm} / \mathrm{kJ}(P=0.35)$ for 50-KGM $v$. control, and 0.112 (sEM 0.0093$) \mathrm{mm} / \mathrm{kJ}(P<0.01)$ for $100-\mathrm{KGM} v .50-\mathrm{KGM}$. For hunger, the differences in SQ were 0.126 (SEM 0.010$) \mathrm{mm} / \mathrm{kJ}$ $(P<0.01)$ for $100-\mathrm{KGM} v$. control, $0.013($ SEM 0.0098$) \mathrm{mm} / \mathrm{kJ}$ $(P=0.41$ ) for $50-\mathrm{KGM} v$. control, and $0 \cdot 113$ (SEM 0.0098$) \mathrm{mm} / \mathrm{kJ}$ $(P<0.01)$ for $100-\mathrm{KGM} v .50-\mathrm{KGM}$. For fullness, the differences in SQ were $0.113(\operatorname{SEM} 0.012) \mathrm{mm} / \mathrm{kJ}(P<0.01)$ for $100-\mathrm{KGM} v$. control, $0.0218($ SEM 0.012$) \mathrm{mm} / \mathrm{kJ}(P=0 \cdot 17)$ for 50-KGM $v$. control, and 0.0911 (SEM 0.012) mm/kJ ( $P<0.01$ ) for 100-KGM $v$. 50KGM. For prospective food consumption, the differences in SQ were $0 \cdot 123$ (SEM 0.0098) mm/kJ $(P<0 \cdot 01)$ for 100-KGM $v$. control, $0.0124($ sem 0.0098$) \mathrm{mm} / \mathrm{kJ}(P=0.42)$ for 50-KGM $v$. control, and $0 \cdot 111(\mathrm{sem} 0.0098) \mathrm{mm} / \mathrm{kJ}(P<0 \cdot 01)$ for 100-KGM $v$. 50-KGM. For the CS, the differences in SQ were 0.129 (SEM 0.0098$) \mathrm{mm} / \mathrm{kJ}$ $(P<0.01)$ for $100-$ KGM $v$. control, 0.0139 (SEM 0.0098$) \mathrm{mm} / \mathrm{kJ}$ $(P=0.35)$ for $50-K G M v$. control, and 0.115 (SEM 0.0098$) \mathrm{mm} / \mathrm{kJ}$ $(P<0 \cdot 01)$ for $100-\mathrm{KGM} v$. 50-KGM. 
Table 2. Mean ratings of satiety measurements over $90 \mathrm{~min}$, palatability, and time to consume preload in sixteen healthy participants (Mean values with their standard errors)

\begin{tabular}{|c|c|c|c|c|c|c|}
\hline \multirow[b]{2}{*}{ Satiety } & \multicolumn{2}{|c|}{ Control } & \multicolumn{2}{|c|}{$50-K G M$} & \multicolumn{2}{|c|}{ 100-KGM } \\
\hline & Mean & SEM & Mean & SEM & Mean & SEM \\
\hline \multicolumn{7}{|l|}{ Mean satiety (mm) } \\
\hline Desire to Eat & 39 & 4 & 39 & 4 & 47 & 4 \\
\hline Hunger & $37^{a}$ & 4 & $38^{a, b}$ & 4 & $47^{\mathrm{b}}$ & 4 \\
\hline Fullness & 55 & 5 & 56 & 5 & 46 & 5 \\
\hline Prospective consumption & $39^{a, b}$ & 4 & $38^{a}$ & 4 & $47^{b}$ & 4 \\
\hline Composite score & 40 & 4 & 40 & 4 & 49 & 4 \\
\hline \multicolumn{7}{|l|}{ Mean satiety quotient (mm/kJ) } \\
\hline Desire to eat & $0.022^{a}$ & 0.007 & $0.035^{a}$ & 0.007 & $0.147^{\mathrm{b}}$ & 0.007 \\
\hline Hunger & $0.022^{a}$ & 0.007 & $0.035^{a}$ & 0.007 & $0.148^{b}$ & 0.007 \\
\hline Fullness & $0.029^{a}$ & 0.010 & $0.051^{a}$ & 0.010 & $0.142^{b}$ & 0.010 \\
\hline Prospective consumption & $0.022^{a}$ & 0.007 & $0.034^{a}$ & 0.007 & $0.145^{\mathrm{b}}$ & 0.007 \\
\hline Composite score & $0.023^{a}$ & 0.007 & $0.037^{a}$ & 0.007 & $0.152^{b}$ & 0.007 \\
\hline \multicolumn{7}{|l|}{ AUC after $90 \mathrm{~min}\left(\mathrm{~mm} \times \mathrm{min}^{-1}\right)$} \\
\hline Desire to eat & 3123 & 376 & 3113 & 375 & 3909 & 375 \\
\hline Hunger & $3009^{a}$ & 394 & $3109^{a, b}$ & 389 & $3954^{b}$ & 393 \\
\hline Fullness & $5257^{\mathrm{a}, \mathrm{b}}$ & 452 & $5429^{a}$ & 454 & $4423^{b}$ & 455 \\
\hline Prospective consumption & $3229^{a, b}$ & 374 & $3046^{a}$ & 374 & $3909^{b}$ & 373 \\
\hline Composite score & 3284 & 386 & 3234 & 384 & 4054 & 386 \\
\hline \multicolumn{7}{|l|}{ Other measurements } \\
\hline Palatability (mm) & 71.5 & 7 & 62.9 & 7 & $56 \cdot 7$ & 7 \\
\hline Time to consume preload (min) & $13 \cdot 2$ & 1 & 11 & 1 & 11.9 & 1 \\
\hline
\end{tabular}

a,b Mean values within a row with unlike superscript letters were significantly different $(P<0.05)$.

Table 3. Presence of gastrointestinal symptoms over 90 min in sixteen healthy participants

\begin{tabular}{lccc}
\hline Gastrointestinal symptoms & Control & 50-KGM & 100-KGM \\
\hline Bloating & 3 & 2 & 5 \\
Belching & 1 & 3 & 2 \\
Dizziness & 0 & 0 & 1 \\
Vomiting & 0 & 0 & 0 \\
Flatulence & 1 & 2 & 3 \\
\hline
\end{tabular}

\section{Other measures}

Palatability ratings between the preloads (100-KGM $v$. control: -14.8 (SEM 8.3 ) mm, $P=0.19$; 50-KGM $v$. control: -8.6 (SEM 8.3 ) mm, $P=0.56$; 100-KGM $v$. 50-KGM: $-6 \cdot 2$ (SEM 8.3) $\mathrm{mm}$, $P=0.73$ ) and the time taken to consume the preloads (100-KGM $v$. control: -1.3 (SEM 1.6) min, $P=0.72 ; 50-\mathrm{KGM} v$. control: $-2 \cdot 1$ (SEM 1.6 ) $\mathrm{min}, P=0 \cdot 41 ; 100-\mathrm{KGM} v$. 50-KGM: $0 \cdot 8$ (sEM 1.6) $\min , P=0.87$ ) were not significantly different (Table 2 ). All preload interventions were well tolerated. The frequency of gastrointestinal symptoms was not statistically different between preloads (Table 3).

\section{Discussion}

To our knowledge, the present study is the first to investigate substituting KGM-gel food analogues into a meal on satiety and subsequent food intake. KGM-gels are made by dispersing a small amount of KGM fibre into water and adding a mild alkalizing agent to induce gelation. As a result, the composition of KGM-gels is approximately $97 \%$ water, giving it a very low ED. We hypothesised that substituting energy-dense pasta with KGM-gel noodles would lead to greater energy compensation in a dose-dependent manner at the subsequent meal due to the large differences in energy and macronutrient content between the preloads. Satiety was not different between the control and 50-KGM, but 100-KGM significantly increased hunger when compared with control and lowered fullness when compared with the 50-KGM. Contrary to our hypothesis, no differences in subsequent food intake at 90 min were detected amongst all preloads. This led to an overall reduction in cumulative energy intake across the treatment period.

One possible explanation for the comparable subsequent energy intake, despite the energy disparity between the preloads is the amount, type, and physical form of dietary fibre present among the preloads. Several reviews have suggested that increased fibre intake is associated with greater satiety and reduced energy intake when provided in an isoenergetic setting ${ }^{(15-17)}$. In particular, viscous soluble fibres appear to be the most effective as they can increase the viscosity of the digestive contents to promote satiety by lowering the gastric emptying rate and modifying nutrient kinetics ${ }^{(4)}$. In a previous study using a similar preloading design, we demonstrated that a $5 \mathrm{~g}$ mixture of high viscosity fibres containing KGM in a powdered form did not affect satiety, but modestly reduced energy intake by $418 \mathrm{~kJ}(100 \mathrm{kcal})$ at $90 \mathrm{~min}$ compared with an insoluble fibre control when provided in energy-matched preloads ${ }^{(13)}$. In contrast, gelled fibres similar to those presently used may exert their satiating effects through different mechanisms, as the viscosity and gelation occurs outside of the stomach. Evidence from other gel-forming fibres, such as pectin and agar, suggest that fibre-gels can increase subjective ratings of fullness without reductions in subsequent energy intake ${ }^{(18,19)}$. 
However, the preloads used in other fibre-gel studies are often energy-matched; thus an energy-deficient fibre-gel such as that used presently may not produce the same satiety effect.

Matching for volume between the preloads may have also contributed to comparable energy intakes during the subsequent meal. Food volume has been observed to be an independent predictor of short-term energy intake in studies of similar design. Increasing meal volume leads to greater gastric distention that triggers afferent vagal signals that promote satiety $^{(20)}$. This claim is supported by studies investigating satiety and food intake after ED manipulations in energymatched preloads and from intragastric infusion studies that bypass oro-sensory cues of satiety ${ }^{(21-23)}$. Although this may explain the comparable energy intakes, it is insufficient to explain the differences in satiety observed after 100-KGM.

Investigations utilising MRI have reported greater delays in gastric emptying by increasing the energy content of a meal by as little as $418 \mathrm{~kJ}$ ( $100 \mathrm{kcal})$, regardless of the volume administered $^{(24)}$. Delays in gastric emptying are consistently associated with increased satiety and suggest that the low satiety response presently observed is driven by the lack of macronutrients rather than the volume of the preloads. This may explain the significantly lower satiety rating of the 100-KGM preload compared with the other preloads of approximately $8-10 \mathrm{~mm}$, which is clinically meaningful for appetite regulation ${ }^{(10)}$. In this context, it would be more appropriate to assess the satiating capacity of the preloads per energy intake than the absolute effect on satiety, as the KGM-gel noodles have almost no energy content and energy intake is the primary concern for weight management ${ }^{(12)}$. The present study showed that the SQ, which assesses the change in satiety as a function of energy consumed, was significantly higher for 100-KGM than control or $50-K G M$. This suggests that satiety increases at a faster rate for $100-\mathrm{KGM}$ than the other preloads when energy intake is matched. Although the evidence on the clinical importance of the SQ is sparse, the SQ for fullness has been suggested to predict energy intake in free-living individuals ${ }^{(1,25)}$.

It has also been proposed that firm fibre-gels, such as KGMgel, may possess unique properties that can assist in satiety and energy regulation. Marciani et $a l^{(8)}$ used MRI on agar fibre-gels of varying strengths and found that a firm gel exceeding $0.65 \mathrm{~N}$ in facture strength was retained in the stomach longer and was correlated with a greater feeling of fullness compared with fibre-gels of a lower strength. The authors concluded that $0.65 \mathrm{~N}$ likely exceed the force exerted during mechanical digestion, leading to incomplete gel digestion and greater retention within the stomach. This can increase gastric distention and is likely a unique property of certain fibre-gels as most foods are susceptible to acidic degradation, reducing firmness. Although the strength of the KGM-gels in the present study was not directly measured, data on commercially prepared KGM-gels report a strength ranging between $0 \cdot 8-1 \cdot 6 \mathrm{~N}$, which may explain the lack of energy compensation ${ }^{(26,27)}$. Alternatively, this high gel strength may have altered the oro-sensory attributes between the preloads, leading to greater oral exposure that affects satiety and food intake ${ }^{(15,28)}$. However, this effect in the present study may be small as palatability and mean eating time between preloads were not significantly different.
As the energy intakes at the subsequent meal were similar, the energy deficit between the preloads was maintained when the cumulative energy intake was calculated (approximately $841 \mathrm{~kJ}$ (201 kcal) for 50-KGM and approximately $1761 \mathrm{~kJ}$ ( $421 \mathrm{kcal}$ ) for 100-KGM). Comparing across various preloading methods, the cumulative reductions observed between 100-KGM and control preloads were higher than those seen with viscous dietary fibre supplementation of higher meal volumes, or preloads with a similar volume and ED (approximately $1.26 \mathrm{~kJ} / \mathrm{g}(0.3 \mathrm{kcal} / \mathrm{g}$ ) in approximately $300 \mathrm{ml}$ ) achieved through fat reduction or increased vegetable consumption ${ }^{(13,29)}$. In the form currently administered in the study, the noodle shape allows for discretionary replacement of a commonly consumed high-carbohydrate food to decrease mealtime energy intake. Taking into consideration that palatability was not significantly different between the preloads, KGM-gels may potentially be a dietary tool to reduce daily energy intake without affecting the type or amount of consumed, which may be a key factor to maintaining compliance in weight management diets.

Based on the present results, it is unclear whether the energetic deficit introduced from the preloads will be sustained beyond the subsequent meal. A reduction in energy intake introduced by low ED foods have been shown to be sustained over several days in healthy individuals ${ }^{(30)}$. Repeated consumption of fibre-gels may have a similar effect but available clinical evidence is sparse. One study supplemented pectin fibre-gels for $15 \mathrm{~d}$ and only observed a modest increase in fullness with no reduction in overall energy intake ${ }^{(31)}$. However, the pectin gel was energy matched with the control and was likely more susceptible to mechanical digestion, suggested by the low gel strength of approximately $0.36 \mathrm{~N}$ reported in the study. Alternatively, the dietary fibres found in fibre-gels may increase production of SCFA through fermentation by the gut microbiota, which can influence satiety hormones such as PYY and GLP- ${ }^{(32)}$. As KGM-gels have been shown to be fermentable in vitro with a profile similar to the un-gelled form, KGM-gels have the potential for long term energy management given the fermentation profile and high gel strength ${ }^{(33-35)}$. Additional benefits of extended KGM consumption may include reductions in LDL-cholesterol, systolic BP, and improvements in glycaemic control in individuals with T2DM, metabolic syndrome and healthy individuals ${ }^{(36-39)}$. However, these metabolic benefits have only been attributed to the viscous, powdered form and future studies should investigate whether these benefits extend to KGM-gels.

Several limitations to the present study should be acknowledged. First, the sample size calculation was based on a large difference in energy content $(628 \mathrm{~kJ}$ ( $150 \mathrm{kcal}))$ as we hypothesised that individuals would compensate for the missing energy content from KGM-gels. As the study was only powered to assess this difference, detection of smaller differences and confidence in secondary outcomes, such as satiety and palatability, was limited by the small sample size of the study. Second, while the dessert ad libitum meal was chosen to represent a commonly consumed snack food, the sweet taste and high palatability may have promoted overconsumption and overrode satiety signals in the control and 50-KGM preload. Although a high palatability food has been 
observed to increase ad libitum energy intake beyond habitual amounts, this wider range of energy intake is suggested to be more sensitive to satiety manipulation and more applicable in a controlled setting ${ }^{(40)}$. Likewise, in similarly palatable meals, difference in ad libitum energy intake between sweet and savoury foods appears to be negligible in healthy individuals, suggesting that the use of a dessert meal may not impact the present findings to a significant degree ${ }^{(41)}$. Third, a negative control was not used to determine ad libitum food intake in the absence of a preload ${ }^{(10)}$. Although several studies have reported reduced ad libitum food intake after preloading, the addition of the preload energy content resulted in higher energy intake than the no preload condition. However, as the KGM-gel contributes negligible energy content to the preload, the total energy intake of the 100-KGM condition is unlikely to exceed a negative control. Lastly, information on dietary restraint was not collected, limiting the interpretation of the subsequent meal, as restrained eaters would be less likely to eat to satiation.

\section{Conclusions}

In conclusion, KGM-gel noodle substitution resulted in a dose-response reduction of cumulative energy intake without altering meal palatability. Due to the soluble fibre content, very low ED, and its ability to replace common high-carbohydrate foods such as pasta without changing meal volume, partial substitution of KGM-gels noodles holds great promise for satiety and food intake regulation and may potentially introduce a new tool for body weight regulation.

\section{Acknowledgements}

The authors would like to thank Johnny Fung from Wellbond Import Export Inc. for the donation of the konjac glucomannan study product.

V. V. currently holds grant support for dietary fibre and ginseng research from the Canadian Diabetes Association and for ginseng research from the National Institute of Horticultural \& Herbal Science, RDA, Korea. Wellbond Import Export Inc. donated the study material. Wellbond Import Export Inc. had no role in the design, analysis or writing of this article.

E. J.: acquired the data, conducted the research, and analysed the data; F. A.-Y.: analysed the data and drafted the manuscript; V. V.: had primary responsibility for the final content; A. L. J., A. Z., H. V. T. H. and V. V.: critically revised the manuscript for important intellectual content. All authors read and approved the final manuscript.

V. V. holds an American (no. 7,326,404 B2) and Canadian (no. $2,410,556$ ) patent for use of a powdered viscous fibre blend in diabetes, metabolic syndrome and cholesterol lowering. At the time of the study, V. V. was a partial owner of Glycemic Index Laboratories (Toronto, ON., Canada) and has since retired from the organisation (April, 2015); A. L. J. is a VP and partial owner of Glycemic Index Laboratories, a clinical research organisation. F. A.-Y. is a contract research assistant for Glycemic Index Laboratories. All other authors have no conflicts of interest to declare.

\section{References}

1. Drapeau V, King N, Hetherington M, et al. (2007) Appetite sensations and satiety quotient: predictors of energy intake and weight loss. Appetite 48, 159-166.

2. Rogers PJ \& Brunstrom JM (2016) Appetite and energy balancing. Physiol Behav 164, 465-471.

3. Almiron-Roig E, Palla L, Guest K, et al. (2013) Factors that determine energy compensation: a systematic review of preload studies. Nutr Rev 71, 458-473.

4. Kristensen M \& Jensen MG (2011) Dietary fibres in the regulation of appetite and food intake. Importance of viscosity. Appetite 56, 65-70.

5. Rolls BJ (2009) The relationship between dietary energy density and energy intake. Physiol Behav 97, 609-615.

6. Chua M, Baldwin TC, Hocking TJ, et al. (2010) Traditional uses and potential health benefits of Amorphophallus konjac K. Koch ex N.E.Br. J Ethnopharmacol 128, 268-278.

7. Parry J (2009) Konjac glucomannan. In Food Stabilisers, Thickeners and Gelling Agents, pp. 198-217 [A Imeson, editor]. Oxford: Wiley-Blackwell.

8. Marciani L, Gowland PA, Fillery-Travis A, et al. (2001) Assessment of antral grinding of a model solid meal with echo-planar imaging. Am J Physiol Gastrointest Liver Physiol 280, G844-G849.

9. Vuksan V, Rogovik AL, Jovanovski E, et al. (2009) Fiber facts: benefits and recommendations for individuals with type 2 diabetes. Curr Diabetes Rep 9, 405-411.

10. Blundell J, de Graaf C, Hulshof T, et al. (2010) Appetite control: methodological aspects of the evaluation of foods. Obes Rev 11, 251-270.

11. Ledikwe JH, Rolls BJ, Smiciklas-Wright H, et al. (2007) Reductions in dietary energy density are associated with weight loss in overweight and obese participants in the PREMIER trial. Am J Clin Nutr 85, 1212-1221.

12. Green SM, Delargy HJ, Joanes D, et al. (1997) A satiety quotient: a formulation to assess the satiating effect of food. Appetite 29, 291-304.

13. Vuksan V, Panahi S, Lyon M, et al. (2009) Viscosity of fiber preloads affects food intake in adolescents. Nutr Metab Cardiovasc Dis 19, 498-503.

14. Samra RA \& Anderson GH (2007) Insoluble cereal fiber reduces appetite and short-term food intake and glycemic response to food consumed $75 \mathrm{~min}$ later by healthy men. Am J Clin Nutr 86, 972-979.

15. Clark MJ \& Slavin JL (2013) The effect of fiber on satiety and food intake: a systematic review. J Am Coll Nutr 32, 200-211.

16. Howarth NC, Saltzman E \& Roberts SB (2001) Dietary fiber and weight regulation. Nutr Rev 59, 129-139.

17. Wanders AJ, van den Borne JJ, de Graaf C, et al. (2011) Effects of dietary fibre on subjective appetite, energy intake and body weight: a systematic review of randomized controlled trials. Obes Rev 12, 724-739.

18. Clegg ME \& Shafat A (2014) The effect of agar jelly on energy expenditure, appetite, gastric emptying and glycaemic response. Eur J Nutr 53, 533-539.

19. Wanders AJ, Feskens EJ, Jonathan MC, et al. (2014) Pectin is not pectin: a randomized trial on the effect of different physicochemical properties of dietary fiber on appetite and energy intake. Physiol Behav 128, 212-219.

20. Ello-Martin JA, Ledikwe JH \& Rolls BJ (2005) The influence of food portion size and energy density on energy intake: implications for weight management. Am J Clin Nutr 82, 236s-241s.

21. Rolls BJ, Bell EA \& Waugh BA (2000) Increasing the volume of a food by incorporating air affects satiety in men. Am J Clin Nutr 72, 361-368. 
22. Rolls BJ, Morris EL \& Roe LS (2002) Portion size of food affects energy intake in normal-weight and overweight men and women. Am J Clin Nutr 76, 1207-1213.

23. Rolls BJ \& Roe LS (2002) Effect of the volume of liquid food infused intragastrically on satiety in women. Physiol Behav $\mathbf{7 6}$, 623-631.

24. Kwiatek MA, Menne D, Steingoetter A, et al. (2009) Effect of meal volume and calorie load on postprandial gastric function and emptying: studies under physiological conditions by combined fiber-optic pressure measurement and MRI. Am J Physiol Gastrointest Liver Physiol 297, G894-G901.

25. Drapeau V, Blundell J, Gallant AR, et al. (2013) Behavioural and metabolic characterisation of the low satiety phenotype. Appetite 70, 67-72.

26. Herranz B, Tovar CA, Solo-de-Zaldívar B, et al. (2012) Effect of alkalis on konjac glucomannan gels for use as potential gelling agents in restructured seafood products. Food Hydrocoll 27, $145-153$.

27. Luo X, He P \& Lin X (2013) The mechanism of sodium hydroxide solution promoting the gelation of Konjac glucomannan (KGM). Food Hydrocoll 30, 92-99.

28. Mela DJ (2006) Eating for pleasure or just wanting to eat? Reconsidering sensory hedonic responses as a driver of obesity. Appetite 47, 10-17.

29. Williams RA, Roe LS \& Rolls BJ (2013) Comparison of three methods to reduce energy density. Effects on daily energy intake. Appetite 66, 75-83.

30. Bell EA, Castellanos VH, Pelkman CL, et al. (1998) Energy density of foods affects energy intake in normal-weight women. Am J Clin Nutr 67, 412-420.

31. Wanders AJ, Mars M, Borgonjen-van den Berg KJ, et al. (2014) Satiety and energy intake after single and repeated exposure to gel-forming dietary fiber: post-ingestive effects. Int J Obes (Lond) 38, 794-800.

32. Chambers ES, Morrison DJ \& Frost G (2015) Control of appetite and energy intake by SCFA: what are the potential underlying mechanisms? Proc Nutr Soc 74, 328-336.
33. Chen HL, Lin YM \& Wang YC (2010) Comparative effects of cellulose and soluble fibers (pectin, konjac glucomannan, inulin) on fecal water toxicity toward Caco-2 cells, fecal bacteria enzymes, bile acid, and short-chain fatty acids. J Agric Food Chem 58, 10277-10281.

34. Chiu YT \& Stewart M (2012) Comparison of konjac glucomannan digestibility and fermentability with other dietary fibers in vitro. J Med Food 15, 120-125.

35. Matsuura Y (1998) Degradation of konjac glucomannan by enzymes in human feces and formation of short-chain fatty acids by intestinal anaerobic bacteria. J Nutr Sci Vitaminol 44, 423-436.

36. Jenkins AL, Jenkins DJ, Wolever TM, et al. (2008) Comparable postprandial glucose reductions with viscous fiber blend enriched biscuits in healthy subjects and patients with diabetes mellitus: acute randomized controlled clinical trial. Croat Med J 49, 772-782.

37. Jenkins DJ, Kendall CW, Axelsen M, et al. (2000) Viscous and nonviscous fibres, nonabsorbable and low glycaemic index carbohydrates, blood lipids and coronary heart disease. Curr Opin Lipidol 11, 49-56.

38. Vuksan V, Jenkins AL, Rogovik AL, et al. (2011) Viscosity rather than quantity of dietary fibre predicts cholesterollowering effect in healthy individuals. $\mathrm{Br} J \mathrm{Nutr} \mathbf{1 0 6}$, 1349-1352.

39. Vuksan V, Jenkins DJ, Spadafora P, et al. (1999) Konjac-mannan (glucomannan) improves glycemia and other associated risk factors for coronary heart disease in type 2 diabetes. A randomized controlled metabolic trial. Diabetes Care 22, 913-919.

40. Deighton K, Frampton J \& Gonzalez JT (2016) Test-meal palatability is associated with overconsumption but better represents preceding changes in appetite in non-obese males. BrJ Nutr 116, 935-943.

41. Griffioen-Roose S, Mars M, Finlayson G, et al. (2009) Satiation due to equally palatable sweet and savory meals does not differ in normal weight young adults. J Nutr 139, 2093-2098. 